\title{
System Identification Methods for Dynamic Testing of Fluid-Film Bearings
}

\author{
JERZY T. SAWICKI \\ Assistant Professor, Department of Mechanical Engineering, Cleveland State University, Cleveland, OH 44115, U.S.A
}

MAURICE L. ADAMS, Jr.

Professor, Dept. of Mechanical \& Aerospace Engineering, Case Western Reserve University, Cleveland, OH 44106, U.S.A

\author{
RUSSELL J. CAPALDI
}

Aerospace Engineer, NASA Lewis Research Center, Cleveland, OH 44135, U.S.A.

\begin{abstract}
There are various system identification approaches typically used to extract the rotordynamic coefficients from simultaneously measured dynamic force and motion signals. Since the coefficient values extracted can vary significantly as a function of the system identification approach used, more attention is needed to treat this issue than is typically included in the rotor dynamics literature. This paper describes system identification and data reduction methods used for extracting rotordynamic coefficients of fluid-film journal bearings. Data is used from a test apparatus incorporating a double-spoolshaft spindle which permits independent control over the journal spin speed and the frequency of an adjustable-magnitude circular orbit, for both forward and backward whirling. For example, a least squares linear regression on the force-displacement equations of the experiment provides only one of the rational approaches to extract the anisotropic rotordynamic coefficients (stiffness, damping and fluid inertia effects). Rotordynamic coefficients are also extracted with both first and second order orbital frequency dependencies. To assess the quality of the measured signals, coherence functions are calculated to relate the time-averaged input motion signals and the time-averaged output force signals.
\end{abstract}

Key Words: System Identification; Fluid-Film Bearings; Rotordynamic Coefficients.

\section{INTRODUCTION}

Tentification of bearing dynamic coefficients in rotorbearing systems has been of great importance because of the difficulty in accurate system modeling and analysis. Lubrication theory offers just a tentative structure for the parametric model. Traditionally, linearized models have been used, see for example Adams and Padovan (1981), based on the assumption of "low" vibration amplitudes, for the steady state running of well-balanced machines. The interactive dynamic radial forces generated by the fluid film in journal bearings and seals, for small perturbations of the journal from the static equilibrium position, are described by the following linear approximation

$$
\begin{aligned}
& \left\{\begin{array}{l}
f_{x} \\
f_{y}
\end{array}\right\}=-\left[\begin{array}{l}
K_{x x} K_{x y} \\
K_{y x} K_{y y}
\end{array}\right]\left\{\begin{array}{l}
x \\
y
\end{array}\right\}-\left[\begin{array}{l}
C_{x x} C_{x y} \\
C_{y x} C_{y y}
\end{array}\right]\left\{\begin{array}{l}
\dot{x} \\
\dot{y}
\end{array}\right\} \\
& -\left[\begin{array}{l}
D_{x x} D_{x y} \\
D_{y x} D_{y y}
\end{array}\right]\left\{\begin{array}{l}
\ddot{x} \\
\ddot{y}
\end{array}\right\}
\end{aligned}
$$

Based on compelling arguments made by Adams (1987), the inertia matrix should be symmetric, i.e., $D_{y x}=D_{x y}$. For experimentally extracted coefficients, this means that when solving for all coefficients, the data reduction algorithm is programmed with the constraint of symmetry on the inertia matrix. A non-zero skew-symmetric component added to an otherwise symmetric inertia matrix surely embodies a non-conservative force field, 
just like the skew-symmetric portion of the stiffness matrix does. However, in contrast to skew-symmetric stiffness, which is held "in check" by the symmetric damping within regimes of dynamic stability, a skewsymmetric inertia effect would have a strength proportional to $\Omega^{2}$. That means the highest frequency modes of the system would be driven unstable, overcoming the positive (symmetric) damping whose strength is only proportional to $\Omega$. This is much the same way that true physical effects embodied in the skew-symmetric stiffness generally destabilize one of the lowest frequency modes, since the stiffness effect varies with $\Omega^{0}$ and the positive damping effect varies with $\Omega$. Basically, to admit skew-symmetry into the inertia matrix does not make physical sense and such does not have to be proven by test or theory. If one blindly uses a curve fit strategy which permits skew-symmetric inertia, they have not shown true skew-symmetric inertia, but in fact have obtained a physically inconsistent model from imperfect test data, as all test data is! For experimentally extracted coefficients, symmetry of the inertia matrix means that when solving for all coefficients, the data reduction algorithm is programmed with the constraint of symmetry on the inertia matrix.

To determine experimentally the rotordynamic coefficients, an appropriate data reduction method must be selected based on the design concept of the test apparatus and excitation signal type. In addition, to extract all the coefficients of the anisotropic model (Eq. (1)), one requires not only non-synchronous excitation (i.e., the frequency of the excitation signal $\Omega$ is entirely independent of the shaft spin speed $\omega$ ), but also two linearly independent sets of displacement-force vector measurements. In most test rigs designed for measurement of rotordynamic coefficients, the test bearing or seal can be rigidly mounted with the excitation applied to the shaft (Nordmann and Schöllhorn, 1980; Childs et al., 1986), or alternatively, the test bearing can be floating on the shaft and excitation is applied on the bearing (Glienicke, 1966-67; Kanki and Kawakami, 1987, Childs and Hale, 1994). Even the second solution does not resemble an inservice bearing, but has the advantage that the static load and the applied force can be easily varied. There are basically two approaches one could undertake: $(i)$ impose dynamical forces and measure displacements (Morton, 1975; Nordmann and Schöllhorn, 1980; Yasuda et al., 1986) or (ii) impose dynamical displacements and measure the forces (Jery et al., 1984; Iwatsubo et al., 1988; Adams et al., 1988). Note that in the second approach, to provide the necessary data to extract the anisotropic-model coefficients one requires a significantly two-dimensional orbit. In the authors' opinion, imposing a circular orbit and measuring the resulting forces provides an experiment closer to applications than imposing the dynamic forces to measure a resulting orbital motion. For data reduction in the frequency domain, the objective is identification of the transfer or response function of the bearing, represented either as mobilities $H_{i j}$ or impedances $Z_{i j}$ (see Rouvas and Childs, 1993). A number of different types of excitations signals have been used, such as single frequency, frequency sweep, random and impact, each having its own advantages and disadvantages. Generally, the choice depends upon the nonlinear behavior of the system and time available for the test event. Muszynska and Bently (1990) showed that the best excitation is a circular periodic function with distinct direction. This type of excitation allows for validation of the basic mechanical impedance model, which would be independent of this directional effect.

This paper describes the data analysis method in which the linearized dynamic model of a four-pocket orifice-compensated hydrostatic journal bearing is identified by using as an excitation input prescribed motion. Presented results illustrate the effectiveness of different data reduction methods, including new frequency dependent approach. It is shown that by suitable selection of data reduction algorithm and linear model structure, it is possible either to justify the hypothesis of frequency independent coefficients or to extract rotordynamic coefficients which are frequency dependent.

\section{TEST APPARATUS}

The apparatus utilized consists of a double-spool shaft spindle and test chamber (Adams et al., 1988). Figure 1 shows a conceptual sketch when set for testing a seal. The inner and outer spindle bearing centerlines are

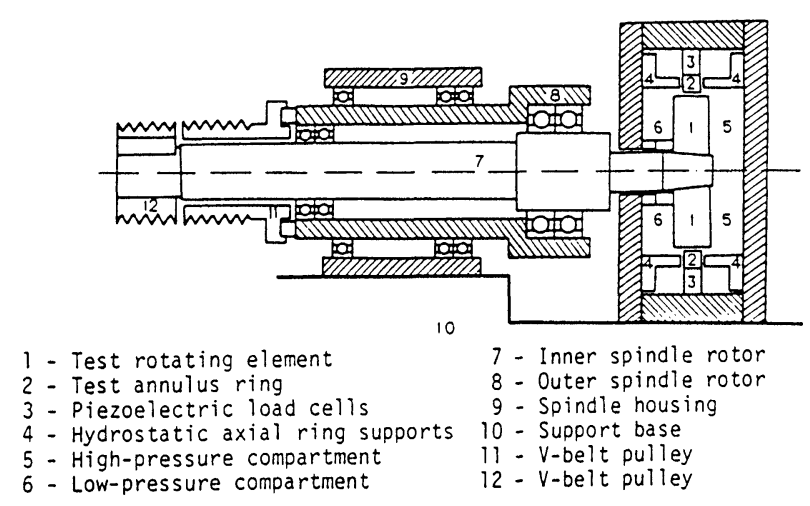

FIGURE 1 Conceptual sketch of test apparatus. 
intentionally manufactured with an adjustable radial offset or eccentricity, so the orbital eccentricity can be accurately adjusted from $0.005 \mathrm{~mm}$ to $1.52 \mathrm{~mm}$. Each spindle shaft is independently driven by a variable speed drive. Rotational speed of the inner shaft is the spin speed, and the rotational speed of the outer shaft provides an independently controlled orbital vibration frequency for both forward and backward whirl. The resulting dynamic force signals exerted upon the tested bearing are then measured by the load measuring system. The test bearing ring assembly is supported in the radial plane by four support links connected to piezoelectric load cells, two in each of the $x$ and $y$ mutually perpendicular directions. The second independent in series load measuring system incorporates a bridge of four small strain gages in each of the four support links, and thereby provides an automatic temperature compensation. In addition to confirming the measurement accuracy of the piezoelectric load cells, the strain gage load cell system also provides the static load measuring capability. Calibration of both load measuring systems is performed simultaneously, in place.

The outer spindle of the double spool shaft, which produces the orbit frequency, is equipped with a timing disk at its drive end. The timing disk contains 360 equally spaced slots which interrupt a light beam from an optical switch, generating external clocking to the data acquisition. The A/D converter is triggered by a second optical switch, one pulse per outer spindle revolution. The clocking is related to the position of the shaft center in the orbit. Eight channels of data are taken to the A/D converter, which include four force signals ( $x$ and $y$ from the two load measuring systems) and four displacement signals ( $x$ and $y$ at each end of the test bearing) using inductance type non-contacting proximity probes by Bently Nevada. Thus, 45 digitized data points are taken from each channel per cycle of orbit. Typically, data is taken for 50 consecutive cycles and time averaged. The eight time-averaged signals are then Fourier Series decomposed to extract the fundamental orbit frequency signal components, which provide the inputs to the test rig governing equations (Eq. 8) to extract the set of rotordynamic coefficients. Due to the fact that only one Sample and Hold circuit exists in the used data acquisition board, there is a one degree time difference between two consecutive channels. Therefore, the signal processing includes phase angle correction of the Fourier coefficients according to the sequence of channels. The signal processing diagram is shown in Figure 2.

Test data for a pocket type hydrostatic journal bearing (Figure 3) was used. The bearing is supplied with a non-detergent motor oil SAE 30 of absolute viscosity

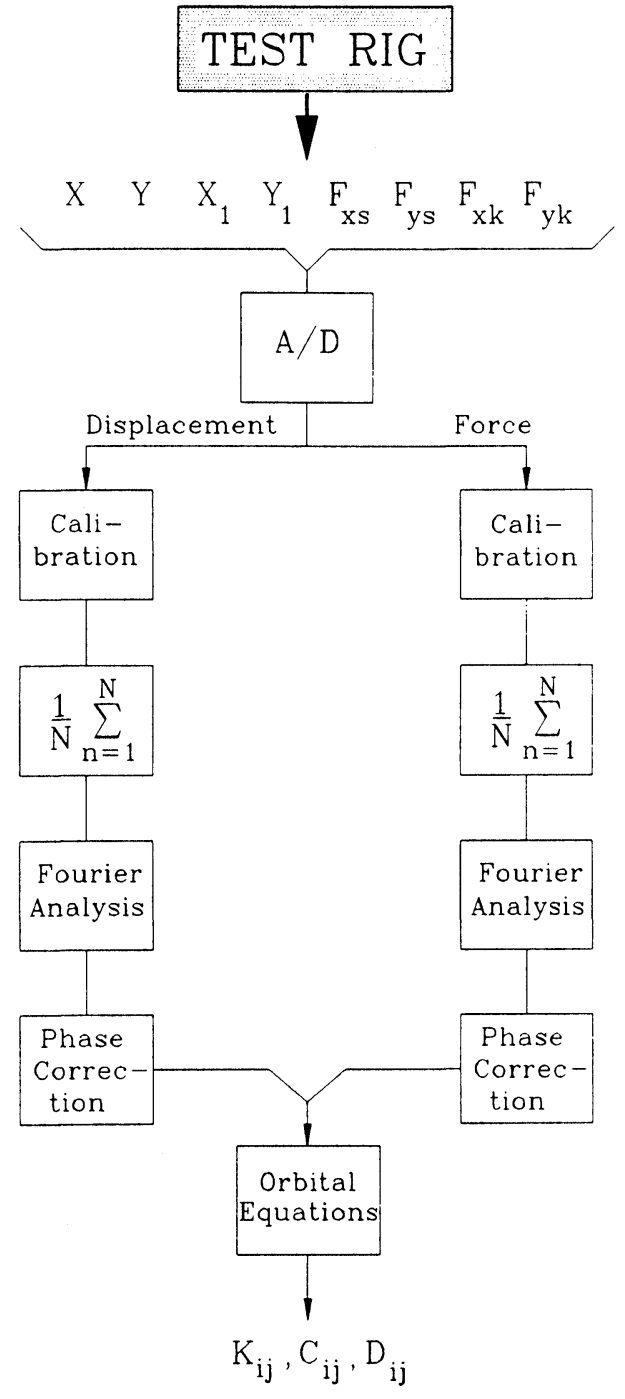

FIGURE 2 Signal processing diagram.

$97987 \cdot 10^{-6} \mathrm{~Pa} \cdot \mathrm{s}$ at $37.78^{\circ} \mathrm{C}$. The lubricant operating temperature is $37.8-43.3^{\circ} \mathrm{C}$. Tests have been made at various bearing operating conditions, including parametric variations of recess pressure, pressure ratio, static eccentricity, vibration orbit radius and rotational speed. The test matrix consists of two speeds (1000 and 2000 $\mathrm{rpm})$, two recess pressures $(0.4826,1.0342 \mathrm{MPa}-70$, $150 \mathrm{psi})$, three pressure ratios $(0.25,0.4,0.55)$, three static eccentricities $(0.0,0.15,0.3)$, three dynamic eccentricities $(0.04,0.096,0.15)$. During all these tests at different speeds, the orbit frequency range is maintained from approximately 0.2 to 1.2 times the shaft speed. Here, data is taken at sufficiently off-synchronous orbit frequencies so that extraneous signals not coherent with the orbit frequency are essentially filtered out. Such 


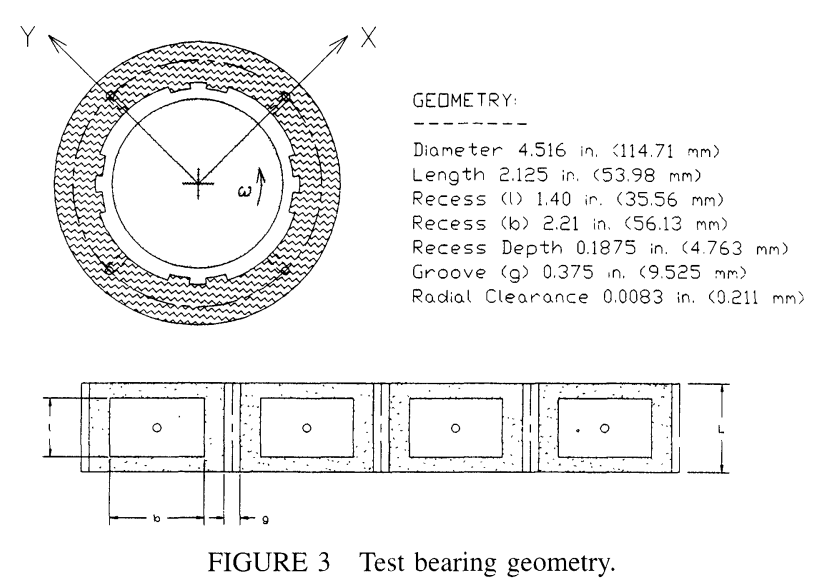

non-coherent signal components include any synchronous-coherent run-out (mechanical and electrical) as well as ball bearing noise, and electrical line frequency interference.

The static eccentricity of the test bearing is accurately adjusted while the apparatus is running. This is done by utilizing the controlled vertical differential thermal expansion between the spindle and test bearing chamber (Sawicki, 1992). Readouts on the relative static eccentricity are extracted from the four displacements signals and provide real-time static position of journal-to-bearing, as well as real-time readout of bearing-to-journal axial misalignment. During the testing, the temperature of the lubricant in the bearing (in the recess and groove) and flow rate is monitored.

\section{METHODOLOGY OF ROTORDYNAMIC COEFFICIENT MEASUREMENT}

To extract the anisotropic coefficients from measured force and displacement signals, the corresponding model of Eq. (1) is assumed and a single-frequency harmonic motion is postulated as follows.

$$
\begin{gathered}
f_{x}=F_{x} e^{i\left(\Omega t+\theta_{s}\right)}, f_{y}=F_{y} e^{i\left(\Omega t+\theta_{3}\right)} \\
x=X e^{i\left(\Omega t+\phi_{s}\right)}, y=Y e^{i\left(\Omega t+\phi_{s}\right)}
\end{gathered}
$$

Substituting Eq. (2) into Eq. (1) gives the following:

$$
\begin{aligned}
& F_{x} e^{i \theta_{i}}=\left(-K_{x x}-i \Omega C_{x x}+\Omega^{2} D_{x x}\right) X e^{i \phi_{i}}+\left(-K_{x y}\right. \\
& \left.-i \Omega C_{x y}+\Omega^{2} D_{x y}\right) Y e^{i \phi_{i}}
\end{aligned}
$$

$$
\begin{aligned}
& F_{y} e^{i \theta_{y}}=\left(-K_{y x}-i \Omega C_{y x}+\Omega^{2} D_{y x}\right) X e^{i \phi_{i}}+\left(-K_{y y}\right. \\
& \left.-i \Omega C_{y y}+\Omega^{2} D_{y y}\right) Y e^{i \phi_{i}}
\end{aligned}
$$

By introducing the following complex notation

$$
\begin{gathered}
\bar{f}_{x}=F_{x} e^{i \theta_{*}}, \bar{f}_{y}=F_{y} e^{i \theta_{1}} \\
\bar{x}=X e^{i \phi_{*},}, \bar{y}=Y e^{i \phi_{3}}
\end{gathered}
$$

Eq. (3) is transformed to the frequency domain and can be written in matrix format as:

$$
\left\{\begin{array}{l}
\bar{f}_{x} \\
\bar{f}_{y}
\end{array}\right\}=-\left[\begin{array}{l}
Z_{x x} Z_{x y} \\
Z_{y x} Z_{y y}
\end{array}\right]\left\{\begin{array}{l}
\bar{x} \\
\bar{y}
\end{array}\right\}
$$

or

$$
\left\{\begin{array}{l}
\bar{x} \\
\bar{y}
\end{array}\right\}=-\left[\begin{array}{l}
H_{x x} H_{x y} \\
H_{y x} H_{y y}
\end{array}\right]\left\{\begin{array}{l}
\bar{f}_{x} \\
\bar{f}_{y}
\end{array}\right\}
$$

Here, $Z_{i j}=K_{i j}+i \Omega C_{i j}-\Omega^{2} D_{i j}$ are impedance functions (or dynamic stiffness functions) based directly on the measurements of $\bar{x}, \bar{y}, \bar{f}_{x}$ and $\bar{f}_{y}$, and $H_{i j}$ are mobilities (where, $\left[H_{i j}\right]=\left[Z_{i j}\right]^{-1}$ ). Also, since the bearing housing is extremely stiff, as is the load measuring system (approximately $9 \times 10^{8} \mathrm{~N} / \mathrm{m}$ ) which attaches the test bearing to the housing, there is no significant d'Alembert force to be taken into account in the force-motion equation.

The test rig has been designed to provide a controlled near perfect circular orbit of radius $R$. Therefore, all phase angles can be referenced to the $x$-component of vibration (i.e., $\phi_{x} \equiv 0$ ) and Eq. (5) yields for each frequency of the forward circular whirl orbit the following relationships:

$$
\begin{aligned}
& \frac{\bar{f}_{x}}{R}=-Z_{x x}+i Z_{x y}=-K_{x x}-\Omega C_{x y}+\Omega^{2} D_{x x}+i\left(K_{x y}\right. \\
& \left.-\Omega C_{x x}-\Omega^{2} D_{x y}\right) \\
& \frac{\bar{f}_{y}}{R}=-Z_{y x}+i Z_{y y}=-K_{y x}-\Omega C_{y y}+\Omega^{2} D_{y x}+i\left(K_{y y}\right. \\
& \left.-\Omega C_{y x}-\Omega^{2} D_{y y}\right)
\end{aligned}
$$

Based on the measurements of $\bar{f}_{x}$ and $\bar{f}_{v}$, the set of 12 rotordynamic coefficients can be determined from a least squares linear regression fit of all frequency data points over the tested frequency range (with shaft speed $\omega$ held constant). To do so, Eq. (7) is segregated by real and imaginary parts to obtain four real equations. Since there are 12 unknowns, experimentally measured data must be 
obtained at a minimum of three discrete orbital frequencies (i.e., $\Omega_{j}, j=1,2,3$ ) for a given operating condition. In the case of the measured dynamic orbit, grouping the real and imaginary components of Eq. (3) or (5), yields the four real, so called here "orbital" equations, as follows.

$$
\begin{aligned}
& F_{x} \cos \theta_{x}=\left[\left(\Omega_{j}^{2} D_{x x}-K_{x x}\right) \cos \phi_{x}+C_{x x} \Omega_{j} \sin \phi_{x}\right] \\
& X+\left[\left(\Omega_{j}^{2} D_{x y}-K_{x y}\right) \cos \phi_{y}+C_{x y} \Omega_{j} \sin \phi_{y}\right] Y \\
& F_{x} \sin \theta_{x}=\left[\left(\Omega_{j}^{2} D_{x x}-K_{x x}\right) \sin \phi_{x}-C_{x x} \Omega_{j} \cos \phi_{x}\right] \\
& X+\left[\left(\Omega_{j}^{2} D_{x y}-K_{x y}\right) \sin \phi_{y}-C_{x y} \Omega_{j} \cos \phi_{y}\right] Y \\
& F_{y} \cos \theta_{y}=\left[\left(\Omega_{j}^{2} D_{y x}-K_{y x}\right) \cos \phi_{x}+C_{y x} \Omega_{j} \sin \phi_{x}\right] \\
& X+\left[\left(\Omega_{j}^{2} D_{y y}-K_{y y}\right) \cos \phi_{y}+C_{y y} \Omega_{j} \sin \phi_{y}\right] Y \\
& F_{y} \sin \theta_{y}=\left[\left(\Omega_{j}^{2} D_{y y}-K_{y x}\right) \sin \phi_{x}-C_{y x} \Omega_{j} \cos \phi_{x}\right] \\
& X+\left[\left(\Omega_{j}^{2} D_{y y}-K_{y y}\right) \sin \phi_{y}-C_{y y} \Omega_{j} \cos \phi_{y}\right] Y
\end{aligned}
$$

The data reduction procedure used for all data presented in this work is based on Eq. (8). However, Eq. (7) produce virtually the same results because the imposed orbit is quite close to an exact circle.

\section{LEAST SQUARES REGRESSION PROCEDURE}

Identification techniques based on least squares regression procedures provide a mathematical tool by which a model can achieve a best fit to experimental data in the sense of minimum-error-squares. They are applicable to both linear and nonlinear problems and facilitate identification of parameters with respect to several inputs simultaneously. The least squares regression technique is applied to solve general orbital equations (Eq. (8)) for rotordynamic coefficients, when a multitude of orbital frequencies are employed. It can be shown, based on Eq. (8), that the relation between the measured and "calculated" force in X or Y-direction, for both load measuring systems, can be expressed as:

$$
\mathbf{y}=v_{1} \mathbf{x}_{1}+v_{2} \mathbf{x}_{2}+\ldots+v_{6} \mathbf{x}_{6}=\sum_{j=1}^{6} v_{j} \mathbf{x}_{j}
$$

where, $\mathbf{y}=\left[F_{p} \cos \theta_{p}, F_{p} \sin \theta_{p}\right]^{T}$ is a vector of measured fluid film forces in $p$-direction $(p=x$ or $y)$, and $\mathbf{x}_{j}=\left[A_{j}\right.$, $\left.B_{j}\right]^{T}$ is a vector of constant coefficients based on the discrete orbital frequencies and Fourier coefficient sets for dynamical displacements, and $v_{j}$ is the set of unknown rotordynamic coefficients corresponding to the fluid force in $x$ - or $y$-direction.

Assuming, that the forces and displacements are measured at $n$ discrete orbital frequencies, Eq. (9) takes the following form

$$
\mathbf{y}=\sum_{j=1}^{6} \mathrm{v}_{j} \mathbf{x}_{i j}, \quad i=1,2, \ldots, n
$$

If vectors $\mathbf{Y}$ and $\mathbf{V}$, and matrix $\mathbf{X}$ are defined as follows

$$
\mathbf{Y}=\left[\mathbf{y}_{1}, \mathbf{y}_{2}, \ldots, \mathbf{y}_{n}\right]^{T}, \quad \mathbf{V}=\left[v_{1}, v_{2}, \ldots, v_{6}\right]^{T}
$$

and

$$
\mathbf{X}=\left[\begin{array}{ccc}
\mathbf{x}_{11} & \cdots & \mathbf{x}_{16} \\
\vdots & \ddots & \vdots \\
\mathbf{x}_{n 1} & \cdots & \mathbf{x}_{n 6}
\end{array}\right]
$$

then, Eq. (10) can be written in a matrix form as:

$$
\mathbf{Y}=\mathbf{X V}
$$

A scalar sum $S$ of the squarred errors in estimation has the form

$$
\mathbf{S}=(\mathbf{Y}-\mathbf{X V})^{T}(\mathbf{Y}-\mathbf{X V})=\operatorname{tr}\left[(\mathbf{Y}-\mathbf{X V})(\mathbf{Y}-\mathbf{X V})^{T}\right]
$$

If $\hat{\mathbf{V}}$ denotes vector of least squares estimates of $\mathbf{V}$, then the following condition must be satisfied (Draper and Smith, 1981),

$$
\left.\frac{\partial S}{\partial \mathbf{V}}\right|_{\mathbf{V}=\hat{\mathbf{V}}}=2\left(\mathbf{X}^{T} \mathbf{X} \hat{\mathbf{V}}-\mathbf{X}^{T} \mathbf{Y}\right)=0
$$

which can be solved for $\hat{\mathbf{V}}$ as

$$
\hat{\mathbf{V}}=\left(\mathbf{X}^{T} \mathbf{X}\right)^{-1} \mathbf{X}^{T} \mathbf{Y}
$$

thus, yielding the linear squares regression identification of rotordynamic coefficients subset $\mathbf{V}$. To have the option of self checking the results, the system of normal equations, Eq. (14), is solved using different numerical algorithms, i.e., Singular Value Decomposition (SVD), Crout's method and iterated improvement of solution (Press et al., 1989). 


\section{NEW FREQUENCY DEPENDENT DATA REDUCTION APPROACH}

There are several approaches to extracting frequency dependent coefficients from experimental data. For example, a localized three-frequency fit, propagated over a frequency range with several frequency data points will produce frequency dependent coefficients to the extent that this improves the fitting of the measurements to the impedance model. Another approach is based on the following imposed relationships:

$$
\begin{aligned}
& K_{i j}=K_{i j}^{(0)}+\Omega^{m} K_{i j}^{(m)} \\
& C_{i j}=C_{i j}^{(0)}+\Omega^{n} C_{i j}^{(n)} \\
& D_{i j}=D_{i j}^{(0)}+\Omega^{p} D_{i j}^{(p)}
\end{aligned}
$$

where $m, n$ and $p$ are positive integers. This representation simply implies that the more rotordynamic coefficients there are, the closer the curve fit comes to passing through all data points. For data reduction mode with damping matrix symmetric and no inertia coefficients ( $K C$-model), this approach gives 14 coefficients, requiring a minimum of 4 orbital test frequencies, while for the mode with $K C D$-model (and inertia matrix symmetric), 22 coefficients requiring a minimum of 6 test frequencies. For a given set of experimental results over some frequency range $\left(\Omega_{\min }, \Omega_{\max }\right)$, one could determine the "optimal" integers $\hat{m}, \hat{n}$ and $\hat{p}$ (or simply an optimal $\hat{m}=$ $\hat{n}=\hat{p}$ ), which produce the best fit, i.e., minimize the fit error based on a least squares approach.

Another approach one could consider is based on the following imposed representation of extracted coefficients:

$$
\begin{aligned}
& K_{i j}=K_{i j}^{(0)}+K_{i j}^{(1)} \Omega+K_{i j}^{(2)} \Omega^{2}+\ldots=\sum_{r=0}^{\infty} K_{i j}^{(r)} \Omega^{r} \\
& C_{i j}=C_{i j}^{(0)}+C_{i j}^{(1)} \Omega+C_{i j}^{(2)} \Omega^{2}+\ldots=\sum_{r=0}^{\infty} C_{i j}^{(r)} \Omega^{r} \\
& D_{i j}=D_{i j}^{(0)}+D_{i j}^{(1)} \Omega+D_{i j}^{(2)} \Omega^{2}+\ldots=\sum_{r=0}^{\infty} D_{i j}^{(r)} \Omega^{r}
\end{aligned}
$$

However, it become immediately obvious that this approach leads to a high number of coefficients and thus is surely impractical. When the terms with powers of $\Omega$ greater than two are truncated, this formulation results in 33 coefficients for $K C D$-model requiring a minimum of 9 test frequencies and 21 coefficients for $K C$-model with a minimum of 6 frequencies. In this work, the $1^{s t}$ and $2^{\text {nd }}$ (i.e., for $r=1$ and 2, respectively) order of frequency dependency is investigated. Recall that the constraint of symmetry in inertia matrix is applied throughout this development, thus the resulting odd number of coefficients. System identification models, including Eq. (16), are just polynomial curve fits in $\Omega$, usually truncated to be consistent with a constant-coefficient $K C D$ model. Equations (16) are logical extension to this, and more important can clearly show for any set of test data the error introduced by the $K C D$ constant-coefficient assumption.

\section{RESULTS AND CONCLUSIONS}

A typical example of comparison between the dynamic load signals of the two independent systems is shown in Fig. 4a. The two signals shown, for the $x$-force component, are time averaged over 50 consecutive cycles, and show very little higher harmonic components. The numerical comparison shows only a $1.8 \%$ difference in amplitude and $0.3^{\circ}$ difference in phase angle. This example is from a series of tests at 10 frequencies with otherwise constant operating conditions. Figure $4 \mathrm{~b}$ shows the filtering of non-coherent interference through time averaging process. With 50 cycles of time averaged data, the total signal is very nearly a perfect sine wave, which is a good indication of dynamic linearity.

To assess the quality of the measured signals, coherence functions have been calculated to relate the timeaveraged $x$ and $y$ input motion signals and $x$ and $y$ time-averaged output force signals from both load measuring systems over the tested frequency range. This gives rise to four coherence functions (Bendat and Piersol, 1993) at each orbital frequency, i.e.,

$$
\begin{aligned}
& \gamma_{x_{i} F_{j}}^{2}\left(f, \Omega_{k}\right)=\frac{\left|G_{x_{i} F_{j}}\left(f, \Omega_{k}\right)\right|^{2}}{G_{x_{x_{i}}}\left(f, \Omega_{k}\right) G_{F_{j} F_{j}}\left(f, \Omega_{k}\right)}, \\
& \text { for } k=1,2, \ldots m ; i, j=x \text { or } y
\end{aligned}
$$

where $m$ is the number of discrete orbital frequencies $\Omega_{\mathrm{k}}$. Spectral density functions are estimated through finite Fourier transforms of the time averaged signals and, for example, the cross-spectral density function at a given orbital frequency $\Omega_{\mathrm{k}}$ is approximated as: 


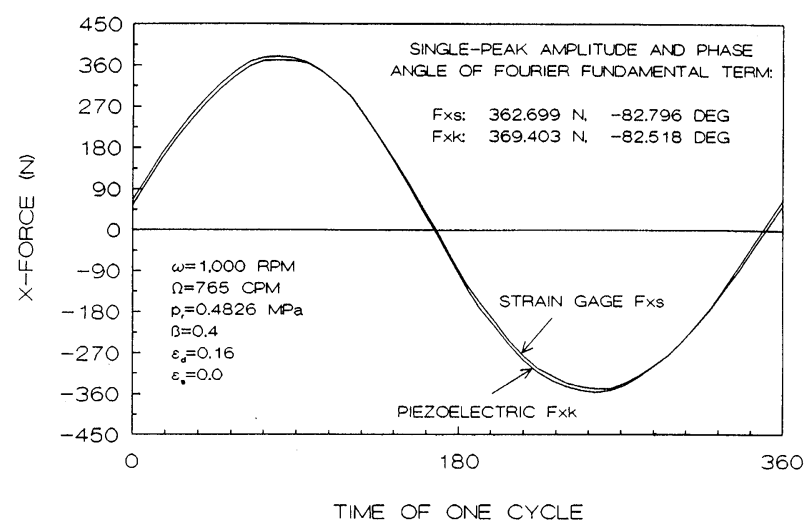

FIGURE 4(a) Time-averaged force signal (50 cycles) from both load systems.

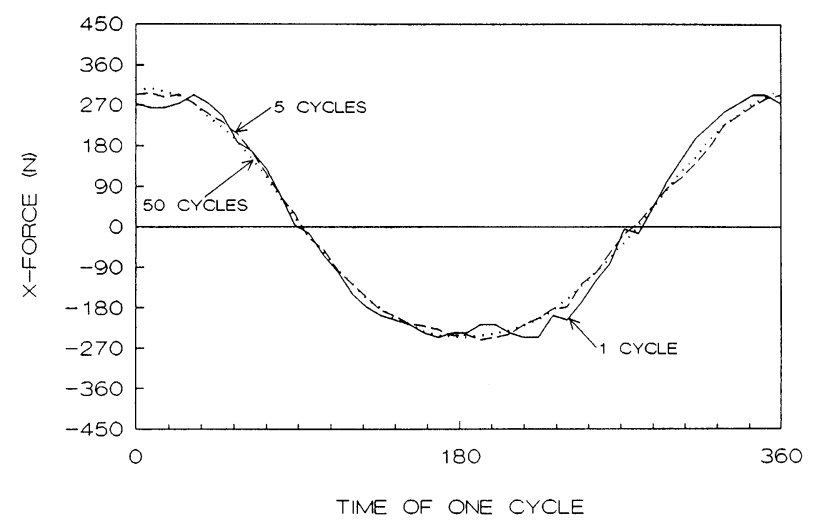

FIGURE 4(b) Time-averaging effect on measured signal (force signal from strain gage load cells).

$G_{x, F_{j}}\left(f, \Omega_{k}\right)=\frac{2}{n_{d}}\left\{\frac{1}{2 \pi} \sum_{s=1}^{n_{d}} X_{i, s}^{*}\left(f, \Omega_{k}, 2 \pi\right) F_{j, s}\left(f, \Omega_{k}, 2 \pi\right)\right\}$,

for $k=1,2, \ldots m ; i, j=x$ or $y$

The typical example of the computed coherence functions $\gamma^{2}$, for force signals measured by piezoelectric load cells, is shown in Table 1 . Note, that all $\gamma^{2}$ are very close to unity at all orbital frequencies, indicating quite "clean" signal measurements and dominant linearity of the apparatus. In this case, the coherence functions also show the effectiveness of the time averaging process. The results are consistent with the obvious fact that the single frequency excitation input motion combined with time averaging over several cycles effectively removes all output signal content not coherent with the input excitation.

Different data reduction modes, including allowed frequency dependence, were investigated to evaluate their effectiveness and influence on the extracted rotor-
TABLE I

Coherence functions between input motion signals and output force signals (piezoelectric load cells): $\omega=1000 \mathbf{R P M}, \varepsilon_{d}=0.04, \varepsilon_{s}=$ $0.0, p_{r}=0.4826 \mathbf{M P a}, \beta=0.25$.

\begin{tabular}{ccccc}
\hline$\Omega / \omega$ & $\gamma_{x . F_{s k}}^{2}$ & $\gamma_{y . F_{x k}}^{2}$ & $\gamma_{x . F_{y k}}^{2}$ & $\gamma_{y \cdot F_{y k}}^{2}$ \\
\hline .247 & .951 & .912 & .941 & .995 \\
.363 & .953 & .928 & .991 & .945 \\
.471 & .996 & .975 & .978 & .968 \\
.569 & .968 & .996 & .925 & .962 \\
.677 & .955 & .960 & .919 & .989 \\
.765 & .982 & .989 & .961 & .939 \\
.871 & .971 & .949 & .963 & .956 \\
1.041 & .939 & .923 & .973 & .927 \\
1.139 & .988 & .949 & .929 & .940 \\
1.247 & .960 & .966 & .989 & .994 \\
\hline
\end{tabular}

dynamic coefficients. Figure 5 shows the comparison between the measured and reproduced $x$-force amplitudes and phase angles (from piezoelectric load cells) using 7 and 11-coefficient fit with no frequency dependency, 14 and 22-coefficient fit with first order frequency
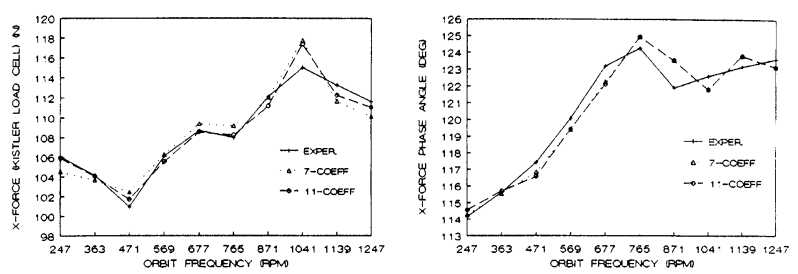

(a) no frequency dependency
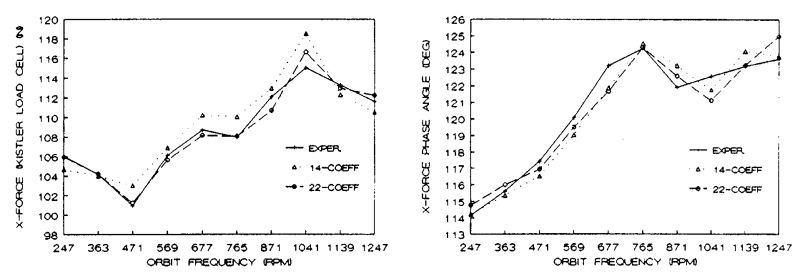

(b) first order frequency dependency
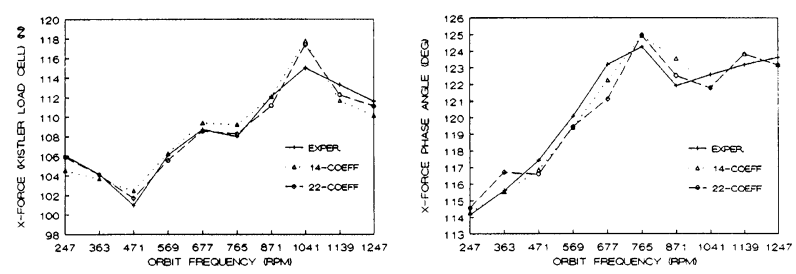

(c) second order frequency dependency

FIGURE 5 Comparison between measured and reproduced X-force amplitudes and phase angles for $\omega=1000 \mathbf{R P M}, \varepsilon_{d}=0.04, \varepsilon_{s}=0.0$, $p_{r}=0.4826 \mathrm{MPa}, \beta=0.25$. (a) no frequency dependency (b) first order frequency dependency (c) second order frequency dependency 
dependency, and 14 and 22-coefficient fit with second order frequency dependency, respectively. The results presented in all those figures support the hypothesis, at least considering the force amplitude components, that the more coefficients there are, the smaller the discrepancies between experimental and recomputed data. Part (a) of Figure 5, created by recomputing the fundamental dynamic force signals using extracted rotordynamic coefficients and the measured fundamental amplitudes and phase angles for the journal displacement signals, shows how good is the hypothesis for frequency independent coefficients. Note, that for this test point, the single-peak amplitudes differ by less than $1.5 \%$, while the phase angles differ by less than $1^{\circ}$. Comparing plots (a), (b) and (c) on Figures 5, the conclusion can be drawn that the 22-coefficient fit with first order frequency dependency matches the experimental data the best.

Rotordynamic coefficients as functions of frequency are shown in Figures 6 and 7. The results indicate that the hypothesis for frequency independent coefficients is quite valid for the bearing tested, particularly for data reduction mode with no inertia matrix and symmetric damping matrix (Figure 7). This is not surprising for an oil film journal bearing, where the fluid flow and there-
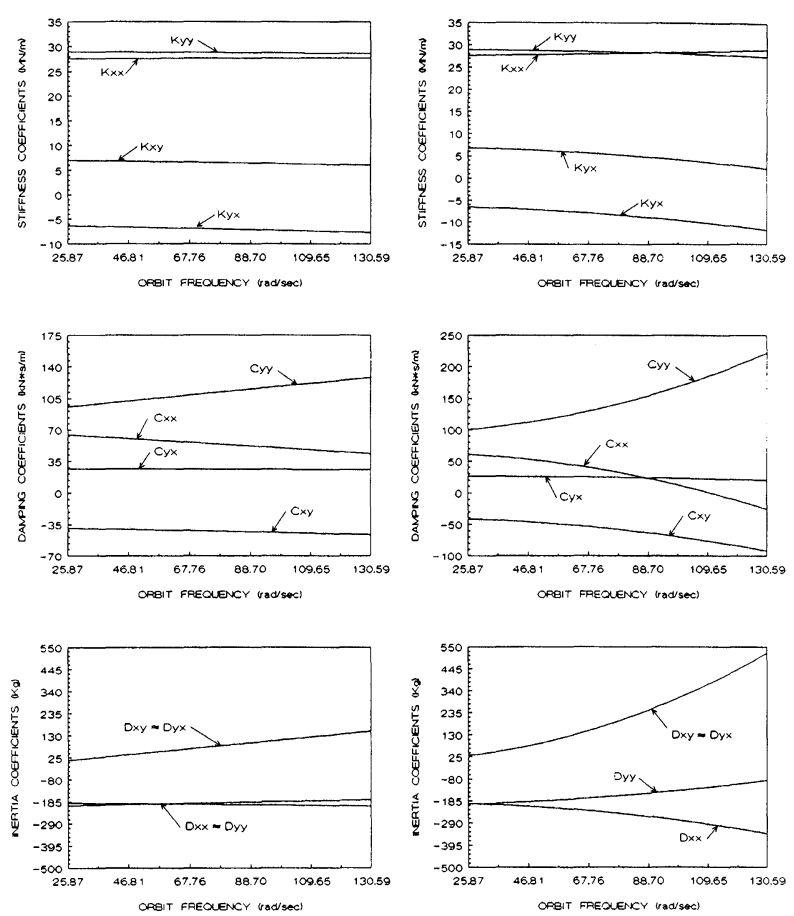

FIGURE 6 Rotordynamic coefficients with inertia matrix symmetric and first order frequency dependency (left column) and second order frequency dependency (right column): $\omega=1000 \mathbf{R P M}, \varepsilon_{d}=0.04, \varepsilon_{s}$ $=0.0, p_{r}=0.4826 \mathrm{MPa}, \beta=0.25$. fore film pressure are dominated by viscous effects. In fact, in the classical Reynolds lubrication equation, fluid inertia is totally neglected. Any skew-symmetric additive to the bearing damping matrix must be a consequence of fluid inertia effects (Adams and Padovan, 1981), therefore the oil bearing damping matrix should be postulated as symmetric (i.e., $C_{x y}=C_{y x}$ ) in this case.

Several approaches to data reduction have been developed and verified, to identify the rotordynamic coefficients of a four-pocket hydrostatic journal bearing with the hybrid effects of journal rotation and thus hydrodynamic effects. For the bearing and operating conditions tested, frequency independent coefficients capture well the dynamic characteristics. However, the assumption of lubricant incompressibility is not an automatic given, first because of the hydrostatic recess volume, and second because of the upstream lubricant/plumbing flexibility. Therefore, the higher order expansion in frequency of rotordynamic coefficients was employed since the compressibility/flexibility issues would be stronger as frequency increases. Presented results suggest that the new concept of series expansion for the rotordynamic coefficients could be beneficial for the study of their frequency dependence as well as for improvement of accuracy in their identification.

$\begin{array}{ll}\text { Nomenclature } & \\ b & \text { recess circumferential length } \\ C & \text { bearing radial clearance } \\ e & \text { journal eccentricity } \\ f & \text { frequency }\end{array}$
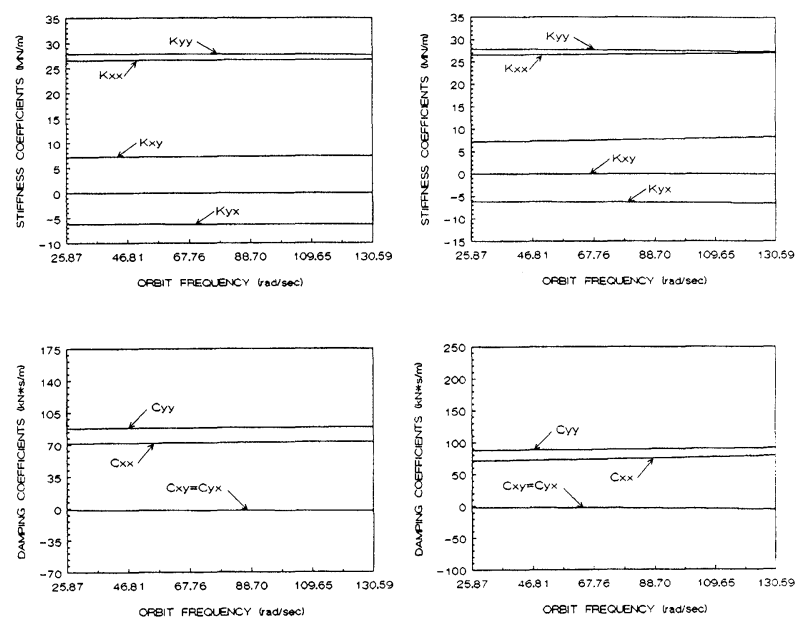

FIGURE 7 Rotordynamic coefficients with no inertia matrix and with first order frequency dependency (left column) and second order frequency dependency (right column): $\omega=1000$ RPM, $\varepsilon_{d}=0.04, \varepsilon_{s}$ $=0.0, p_{r}=0.4826 \mathbf{M P a}, \beta=0.25$. 


$$
\begin{aligned}
& f_{x}, f_{y} \\
& \bar{f}_{x}, \overline{\bar{f}}_{y} \\
& F_{x,}, F_{y} \\
& F_{j . s}\left(f, \Omega_{k}, 2 \pi\right)
\end{aligned}
$$

$g$

$G_{i i}$

$G_{i j}$

$H_{i j}$

$i$

$K_{i j}, C_{i j}, D_{i j}$

$K_{i j}^{(r)}, C_{i j}^{(r)}, D_{i j}^{(r)}$

$L, l$

$n_{d}$

$p_{r}$

$R$

$r$

$S$

$t r$

$\hat{\mathbf{V}}$

$\hat{\mathbf{V}}$

$x, y$

$\bar{x}, \bar{y}$

$X, Y$

$X_{i . s}\left(f, \Omega_{k}, 2 \pi\right)$

$Z_{i j}$

$\beta$

$\theta_{x}, \theta_{y}$

$\phi_{x}, \phi_{y}$

$\gamma^{2}$

$\Omega$

$\omega$

$\varepsilon_{d}$

$\dot{\varepsilon_{s}} \partial x()$

()

(

$($ )* fluid interactive force components complex amplitude of fluid film force components

amplitude of fluid film force components finite Fourier transform over the sth record of fluid film force component $(j=x$ or $y)$ of length $2 \pi$ at orbital frequency $\Omega_{k}$ groove width autospectral density function cross-spectral density function frequency response functions (mobilities) imaginary number $(\sqrt{-1})$ elements of stiffness, damping and inertia matrix, respectively

elements of stiffness, damping and inertia matrix, respectively, associated with $r^{\text {th }}$ power of orbital frequency

bearing and recess axial lengths number of time averaged records recess pressure

bearing radius; also orbit radius order of frequency dependency scalar sum of the squarred errors trace of matrix

vector of rotordynamic coefficients vector of least squares estimates of rotordynamic coefficients

displacement components of shaft complex displacement components of shaft amplitude of displacement components of shaft finite Fourier transform over the sth record of displacement component $(i=x$ or $y$ ) of length $2 \pi$ at orbital frequency $\Omega_{k}$ impedance functions (dynamic stiffness functions)

recess-to-supply pressure ratio

phase of fluid film force components

phase of shaft displacement components

coherence function

vibration orbit frequency

rotational speed

dynamic eccentricity ratio, $R / C$ (orbit radius/ radial clearance)

static eccentricity ratio, $e / C$

partial derivative of ()

time derivative of $(d() / d t)$

second time derivative $\left(d^{2}() / d t^{2}\right)$

conjugate of the complex vector

\section{References}

Adams, M. L., 1987. Insights into Linearized Rotor Dynamics, Part 2, Journal of Sound and Vibration, Vol. 112, pp. 97-110.

Adams, M. L., and Padovan, J., 1981. Insights into Linearized Rotor Dynamics, Journal of Sound and Vibration, Vol. 76, pp. 129-142.

Adams, M. L., Sawicki, J. T., and Capaldi, R. J., 1992. "Experimental Determination of Hydrostatic Journal Bearing Rotordynamic Coefficients," Proceedings of the IMechE Fifth International Conference on Vibration in Rotating Machinery, pp. 365-374.

Adams, M. L., Yang, T., and Pace, S. E., 1988. A Seal Test Facility for the Measurement of Isotropic and Anisotropic Linear Rotordynamic Characteristics, Workshop on Rotordynamic Instability Problems in High Performance Turbomachinery, Texas A\&M University, NASA CP 3026, pp. 253-268.

Bendat, J. S., and Piersol, A. G., 1993. Engineering Applications of Correlation and Spectral Analysis, Second Edition, Wiley-Interscience, New York.

Childs, D. W., Nelson, C. E., Nicks, C., Scharrer, J., Elrod, D., and Hale, K., 1986. Theory versus Experiment for the Rotordynamic Coefficients of Annular Gas Seals: Part 1-Test Facility and Apparatus, ASME Journal of Tribology, Vol. 108, pp. 426-432.

Childs, D., and Hale, K., 1994. A Test Apparatus and Facility to Identify the Rotordynamic Coefficients of High-Speed Hydrostatic Bearings, ASME Journal of Tribology, Vol. 116, pp. 337-344.

Draper, N. R., and Smith, H., 1981. Applied Regression Analysis, Second Edition, Wiley, New York.

Glienicke, J., 1966-67. Experimental Investigation of the Stiffness and Damping Coefficients of Turbine Bearings and their Application to Instability Prediction, Proc. of the Institution of Mechanical Engineers (IMechE), Vol. 181, Part 3B, pp. 116-129.

Iwatsubo, T., Sheng, B. C., and Matsumoto, T., 1988. An Experimental Study on the Static and Dynamic Characteristics of Pump Annular Seals, Workshop on Rotordynamic Instability Problems in High Performance Turbomachinery, Texas A\&M University, NASA CP 3026, pp. 229-252.

Jery, B., Acosta, A. J., Brennen, C. E., and Caughey, T. K., 1984. Hydrodynamic Impeller Stiffness, Damping, and Inertia in the Rotordynamics of Centrifugal Flow Pumps, Workshop on Rotordynamic Instability Problems in High Performance Turbomachinery, Texas A\&M University, NASA CP 2338, pp. 137-160.

Kanki, H., and Kawakami, T., 1987. Experimental Study on the Static and Dynamic Characteristics of Screw Grooved Seals, 11th Biennial Conference on Mechanical Vibration and Noise, Rotating Machinery Dynamics, ASME Publication DE-Vol. 2. pp. 273-278.

Morton, P. G., 1975. The Derivation of Bearing Characteristics by Means of Transient Excitation Applied Directly to a Rotating Shaft, G.E.C. Journal of Science and Technologr, Vol. 42, pp. 43-47.

Muszynska, A., and Bently, D. E., 1990. Frequency-Swept Rotating Input Perturbation Techniques and Identification of the Fluid Force Models in Rotor/Bearing/Seal Systems and Fluid Handling Machines, Journal of Sound and Vibration, Vol. 143, pp. 103-124.

Nordmann, R., Schollhorn, K., 1980. Identification of Stiffness and Damping Coefficients of Journal Bearings by Means of the Impact Method, Proceedings of the IMechE Second International Conference, Vibrations in Rotating Machinery, Paper C285/80, pp. 231-258.

Press, W. H., Flannery, B. H., Teukolsky. S. A., and Vetterling, W. T. 1989. Numerical Recipes. The Art of Scientific Computing, Cambridge University Press.

Rouvas, C., and Childs, D. W., 1993. A Parameter Identification Method for the Rotordynamic Coefficients of a High Reynolds Number Hydrostatic Bearing, ASME Journal of Vibration and Acoustics, Vol. 115, pp. 264-270.

Sawicki, J. T., 1992. Experimental and Theoretical Determination of Hydrostatic/Hybrid Journal Bearing Rotordynamic Coefficients, Ph.D. thesis, Case Western Reserve University, Cleveland, OH.

Yasuda, C., Kanki, H., Ozawa, T., and Kawakami, T., 1986. Application of Random Excitation Technique to Dynamic Characteristics Measurement of Bearing, The International Conference of Rotordynamics, Tokyo, Japan, pp. 61-68. 

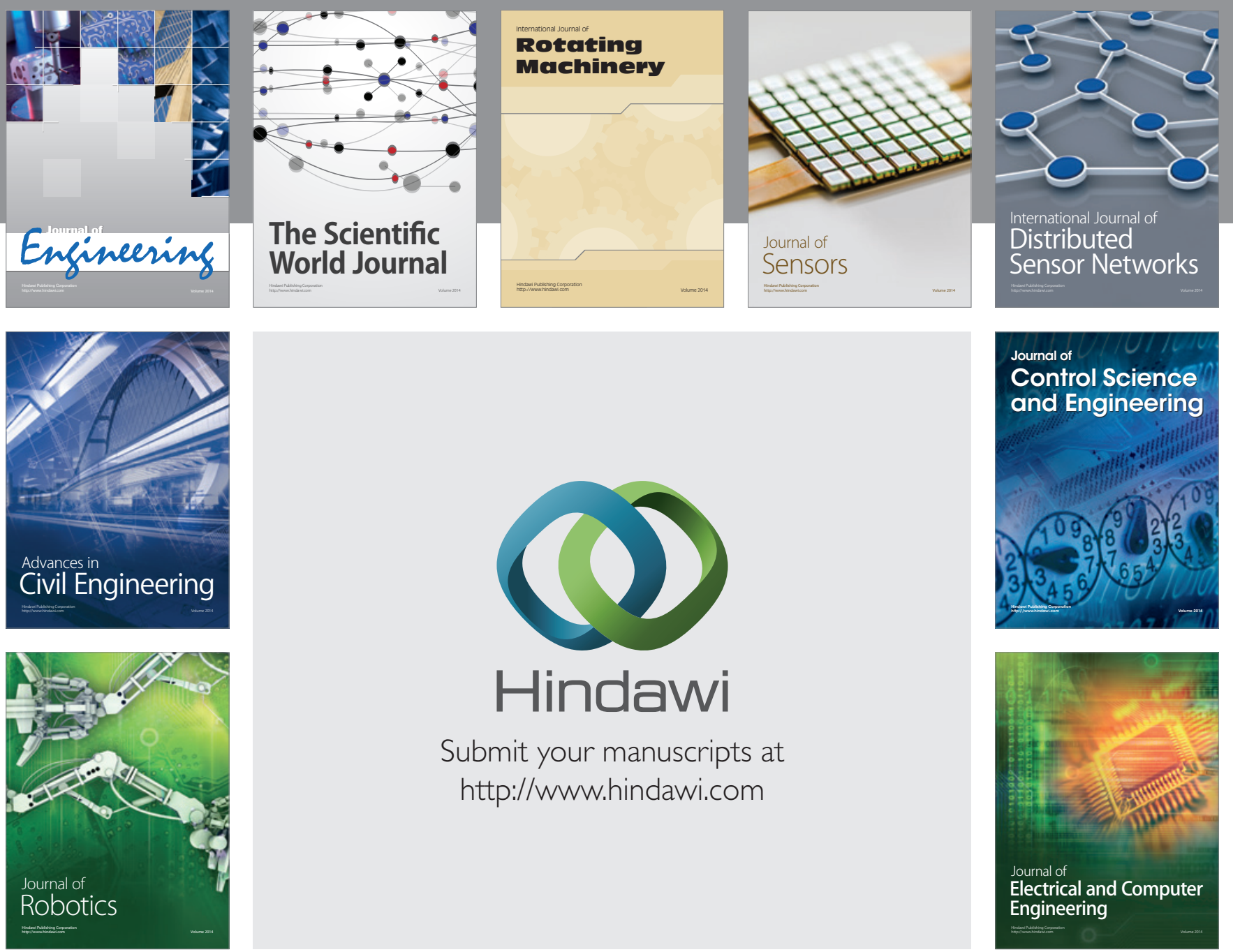

Submit your manuscripts at

http://www.hindawi.com
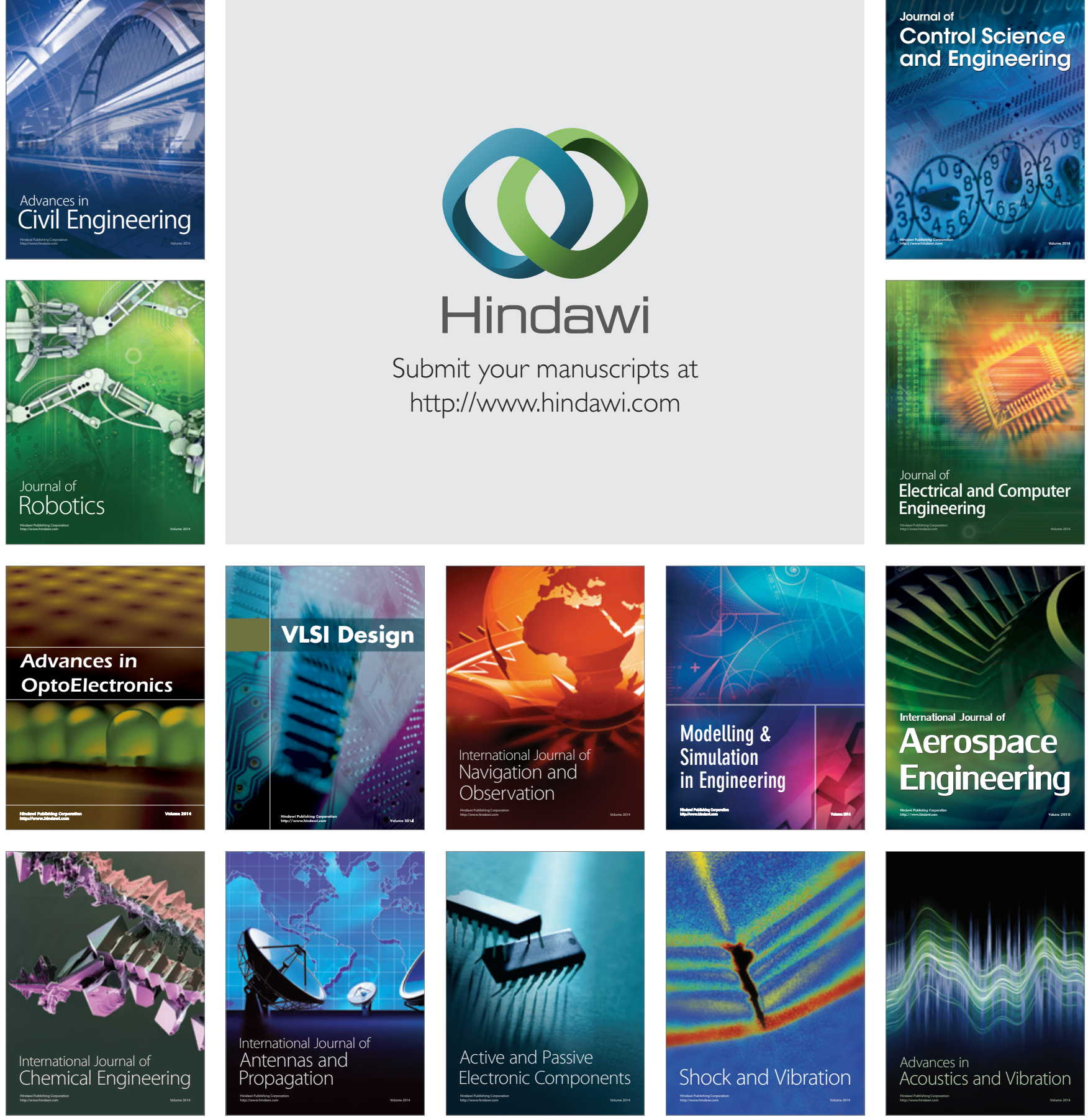